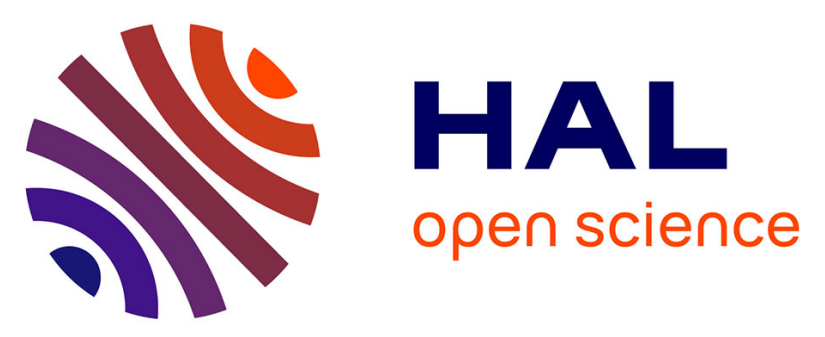

\title{
Network-Based Modeling of Transport Phenomena in Solid and Fluid Phases of Open-Cell Foams: Construction of Graphs
}

\author{
Haithem Louati, Tobias M. Scheuermann, Bernhard Maschke, Marie-Line \\ Zanota, Jérôme Vicente, Paul Kotyczka, Isabelle Pitault
}

\section{To cite this version:}

Haithem Louati, Tobias M. Scheuermann, Bernhard Maschke, Marie-Line Zanota, Jérôme Vicente, et al.. Network-Based Modeling of Transport Phenomena in Solid and Fluid Phases of OpenCell Foams: Construction of Graphs. Advanced Engineering Materials, 2020, 22 (5), pp.1901468. 10.1002/adem.201901468 . hal-02564935

\section{HAL Id: hal-02564935 https://hal.science/hal-02564935}

Submitted on 11 Nov 2020

HAL is a multi-disciplinary open access archive for the deposit and dissemination of scientific research documents, whether they are published or not. The documents may come from teaching and research institutions in France or abroad, or from public or private research centers.
L'archive ouverte pluridisciplinaire HAL, est destinée au dépôt et à la diffusion de documents scientifiques de niveau recherche, publiés ou non, émanant des établissements d'enseignement et de recherche français ou étrangers, des laboratoires publics ou privés. 


\title{
Network based modelling of transport phenomena in solid and fluid phases of open cell foams: construction of graphs
}

\author{
Haithem Louati $^{1 *}$, Tobias Scheuermann ${ }^{2}$, Bernhard Maschke ${ }^{1}$, Marie-Line Zanota ${ }^{3}$, Jerome Vicente ${ }^{4}$, \\ Paul Kotyczka ${ }^{2}$, Isabelle Pitault ${ }^{1}$
}

\author{
${ }^{1}$ Univ. Lyon, Université Claude Bernard Lyon1, CNRS, LAGEPP UMR 5007, 43 boulevard du 11 novembre 1918, F-69100 \\ Villeurbanne, France. \\ ${ }^{2}$ Technical University of Munich, Department of Mechanical Engineering, Chair of Automatic Control, Boltzmannstraße \\ 15, 85748 Garching, Germany. \\ ${ }^{3}$ Univ. Lyon, CNRS, CPE Lyon, UCBL, LGPC UMR 5285, 43 boulevard du 11 novembre 1918, F-69100 Villeurbanne, \\ France. \\ ${ }^{4}$ IUSTI, CNRS UMR 7343, Université de Aix-Marseille, Marseille 13453, France.
}

*Haithem Louati : e-mail haithem.louati@univ-lyon1.fr

\section{$\underline{\text { Highlights }}$}

- Graph representations of the foam topology are obtained using X-ray CT and iMorph.

- Graphs of solid and fluid phases are constructed based on Plateau's laws.

- A coupled graph is constructed for phenomena at interphases

- Discrete conservation laws expressed on graphs and Cell-complex.

\section{$\underline{\text { Abstract }}$}

From 3D X-ray computed images and using iMorph software, two graphs were derived from the solid and fluid networks of open cell foams. The graphs are constructed by applying Plateau's laws, which are satisfied by the foam structure. We have defined two types of graph: (i) the representative graphs of solid and fluid phase networks; The graph of solid phase is, intuitively, constructed by associating vertices to nodes and edges to struts. For the graph of fluid phase, vertices are associated with porous cells and edges to throats at the interface of two cells. (ii) the coupling graph is related to transfer phenomena between the fluid and solid phases. It is constructed by associating effective edges, connecting a vertex of solid to an adjacent vertex of fluid, to the crossed surface of the foam node.

The graphs lead to discrete element representation (topology preserving) with geometrical information of the foam structure. Physical variables can be attributed to the discrete objects called Cell-complex: internal energy to finite volume, heat flux to surface/interface, driving force to edge and temperature to node. This alternative modeling approach based on graphs is promising for heterogeneous systems with large size since it reduces the computational complexity of conventional finite element meshing.

Keywords: Open cell foam, Plateau's laws, X-ray CT, iMorph, Graph, Cell-complex, Modelling.

Acknowledgements: This work was supported by the Agence Nationale de la Recherche - Deutsche Forschungsgemeinschaft (ANR-DFG), project INFIDHEM, ID ANR-16-CE92-0028, Lo 408/22-1. 


\section{List of abbreviations}

\begin{tabular}{|c|c|}
\hline$A$ & Adjacency matrix \\
\hline$A_{i}$ & Heat transfer surface area $\left(\mathrm{m}^{2}\right)$ \\
\hline$C_{p}$ & Heat capacity $\left(J . K^{-1} \cdot \mathrm{kg}^{-1}\right)$ \\
\hline$D$ & Degree Matrix \\
\hline$D_{p}$ & Diameter of an equivalent sphere to a pore volume $(\mathrm{mm})$ \\
\hline$D_{n}$ & Diameter of an equivalent sphere to a node volume $(\mathrm{mm})$ \\
\hline$E$ & Set of edges \\
\hline$E_{b}$ & Set of boundary edges \\
\hline$f$ & Fluid \\
\hline$h$ & Heat transfer coefficient solid-fluid $\left(W \cdot m^{-2} \cdot K^{-1}\right)$ \\
\hline$F$ & Driving force \\
\hline$K$ & Cell-complex / K-complex \\
\hline$\widetilde{K}$ & Dual Cell-complex \\
\hline$L$ & Laplacian matrix \\
\hline$M$ & Incidence matrix \\
\hline$s$ & Solid \\
\hline$S$ & Surface $\left(\mathrm{mm}^{2}\right)$ \\
\hline$S_{n}$ & Average surface of solid node $\left(\mathrm{mm}^{2}\right)$ \\
\hline$S_{s}$ & Average surface area of strut section $\left(\mathrm{mm}^{2}\right)$ \\
\hline$t$ & Time $(s)$ \\
\hline$T$ & Temperature $(K)$ \\
\hline$u$ & Internal energy $(J)$ \\
\hline$v$ & Volume $\left(m^{3}\right)$ \\
\hline$V$ & Set of vertices \\
\hline$V_{b}$ & Boundary vertex \\
\hline$X$-ray $C T$ & $X$-ray computed tomography \\
\hline
\end{tabular}

\section{List of Greek letters}

$\begin{array}{ll}\varepsilon & \text { Porosity } \\ \lambda & \text { Thermal conductivity }\left(W \cdot m^{-1} \cdot K^{-1}\right) \\ \lambda_{e f f} & \text { Effective thermal conductivity }\left(W \cdot m^{-1} \cdot K^{-1}\right) \\ \lambda_{f} & \text { Solid thermal conductivity }\left(W \cdot m^{-1} \cdot K^{-1}\right) \\ \lambda_{s} & \text { Fluid thermal conductivity }\left(W \cdot m^{-1} \cdot K^{-1}\right) \\ \rho & \text { Density }\left(k g . m^{-3}\right) \\ \rho_{\text {rel }} & \text { Relative density } \\ \rho_{p} & \text { Pore density }(\text { ppi) } \\ \phi & \text { Heat flux }\left(W \cdot m^{-2}\right)\end{array}$




\section{Introduction}

Since the early $20^{\text {th }}$ century, a new kind of porous materials (also called foams or sponges) have attracted different industrial sectors (automotive, aerospace, chemical, etc.) and various types of solid foams came into use (Lefebvre et al., 2008). Foams are made up of two phases: solid and gas. The solid phase or the foam matrix is made of interconnected cells forming a solid network including a characteristic void fraction. Foams can be classified into two categories (i) closed cell foams if the gas phase in the porous cells is not connected (ii) open cell foams if the porous cells are interconnected between them through throats. Open cell foams can be described as a network of ligaments along the edges of cells. A typical void volume fraction of open cell foams is in the range of 80\%-97\% (Giani et al., 2005). Different materials have been used to produce foams including polymers such as polyurethane, ceramic and metals (pure or alloy). In this paper, we are interested in metal foams with open cells.

Open metal cell foams have a number of particular properties including a good mechanical resistance despite their lightness, a good thermal resistance, an energy absorption capability, which are of great interest in different applications such as: heat exchangers and heat sinks (Han et al., 2012; Zhao, 2012), implant matrix for medical applications (Müller et al., 2013), thermal energy storage (Zhao et al., 2010), electrical energy storage (Liu et al., 1999). Besides, since pore size can be controlled, this offer other applications such as high-temperature gas and fluid filtration (Ashby et al., 2000). High surface to volume ratio and low pressure drop make metal foams interesting objects to be used instead of monoliths in structured catalytic reactors (Frey et al., 2016; Giani et al., 2005). The properties of metal foams are inherited from materials from which they are made, but also depends upon the process used for manufacturing, the relative density and structure.

The influence of the foam structure on its properties is a topic of intense current studies. For example, the thermal properties of open cell foam are characterised by an effective thermal conductivity, which depends on the structure of foams and their cell orientations; Open cell foams with different morphology or/and geometrical properties are expected to have different thermal coefficients. The determination of the effective thermal conductivity has been approached in different ways in the literature. Many modelling approaches were based on homogeneous structure (Calmidi et al., 1999; Boomsma and Poulikakos, 2001; Bhattachaya et al., 2002). As thermal conductivity is proportional to porosity, the latter is estimated from some averaged geometries calibrated from experiments. However, it has been shown that thermal properties as well as flow properties are influenced by the local morphology of porous and solid networks. It has been reported that the strut orientations and their intersections (lumping of metal) lead to modify the thermal resistance (Bhattachaya, 2002). Moreover, the reduction of the ligament cross section for the same void fraction leads to reduced heat transfer (Brun et al., 2007; Bracconi et al., 2018). Modelling of thermal properties based on the real geometry of foams obtained from X-ray tomography was also reported in the literature. A number of analytical models were developed from the geometrical parameters obtained from X-ray tomography analysis (De Jaeger, 2011; Mendes et al., 2014; Kumar, et al., 2014; Amani et al., 2018). Since geometry of foams are irregular and anisotropic, a particular structure was used in some studies for sake of simplification. De Jaeger et al. have used different in house manufactured foams with controlled characteristics (e.g. no lump formation at nodes) and they proposed a geometrical model based on periodic unit-cell construction. Kumar et al. have considered a periodic isotropic structure (Kelvin cell) to be analysed and to determine the geometrical parameters for numerical simulations. Mendes et al. and Amani et al. have meshed the 3D CT images with continuum finite elements in order to perform simulation to predict thermal conduction in open cell foam. They affirmed that it is important considering a real geometry of foam in order to obtain accurate results of effective thermal conductivity.

We suggest in this paper a new approach based on the graphical representation of the foam in order to derive the dynamical model of the heat transfer. This approach offers a simplification to the exhaustive continuum meshing of the foam geometry but in the same time preserve the morphological data of samples. Thereby, it allows for considering models of large volumes of open cell foam. In order to construct the graph, the solid structure needs to satisfy certain criteria, which are discussed in this paper. We present a methodology to obtain the graphical model starting from X-ray tomography scan and image processing using the open software iMorph $\odot$ (Brun et al. 2008). This paper is structured in the following way. In the first section, we detail the manufacturing processes of metallic open cell foam in order to understand the obtained structures and their properties. Then, we show how their geometrical properties can be processed from images obtained by X-ray tomography and using iMorph. In particular, we recall how the graphs representing the topology of the solid and fluid phases are constructed. The second section gives a brief introduction to the Cell method (Tonti, 1976): a computational method for modelling of heterogeneous media on discrete topological representation where 
physical quantities are referred to elements of graphs called Cell-complex. In the third section, two samples of open cell foams are analysed using iMorph in order to generate the graphs of the two phases and their pertinence is discussed. Indeed, the accuracy of graphs depends upon the CT scan resolution and the image processing methods used to treat the complex structure.

\section{Open cell foam}
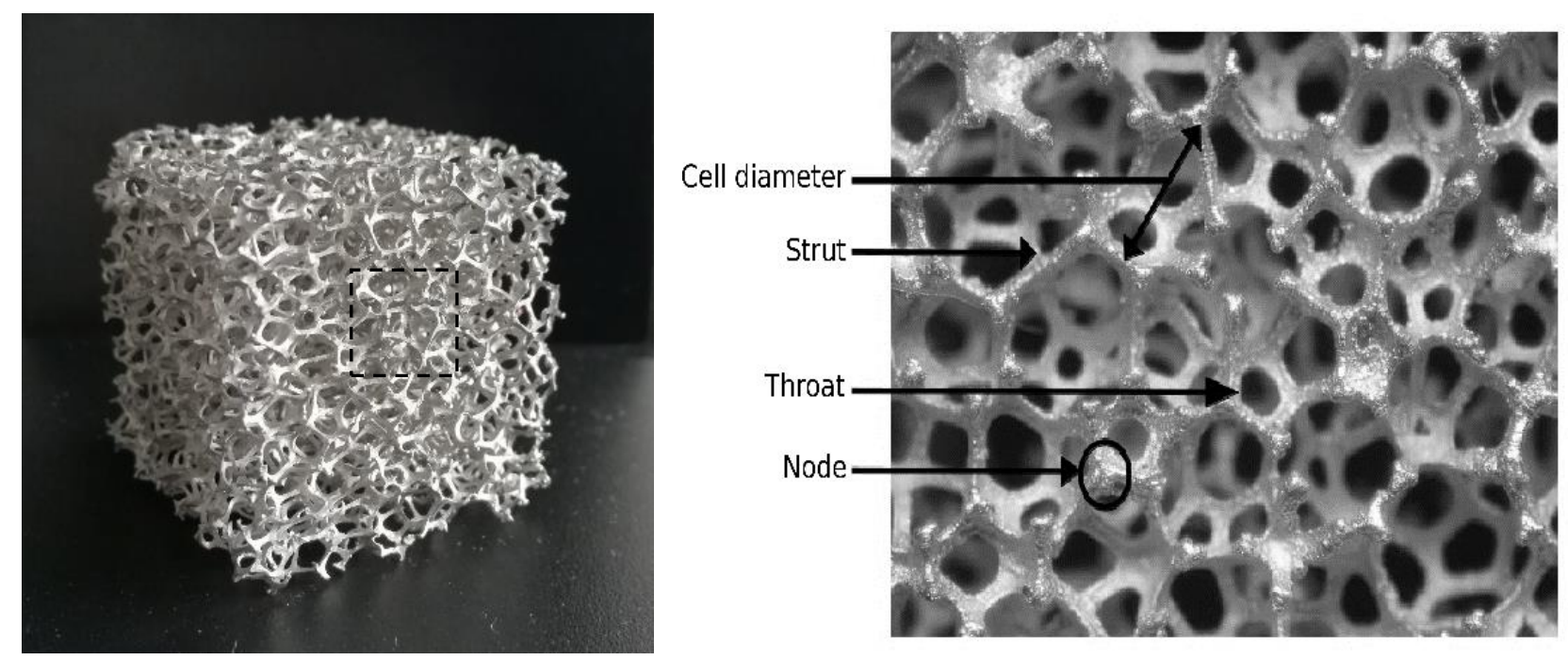

Fig1. Stochastic structure of a polyhedral Aluminium open cell foam.

\subsection{Manufacturing of open cell-foam}

Different techniques have been developed to manufacture metallic cell foams. These techniques can be classified by considering the state of the material to be processed, namely vapour, liquid, powdered, or ionised solution. Thus, methods include vapour deposition, direct blowing in a molten metal, casting liquid metal, sintering of hollow spheres, slurry foaming, electrochemical deposition, etc. (Banhart, 2001). Some of these techniques are more adapted for open cell foams manufacturing than others. In this section, we give a brief introduction to the commonly used techniques for open cell metal foams manufacturing.

- Electro-deposition and vapour deposition methods: The metallic open foams are formed by using a polymer foam template (e.g. polyurethane). The metal is deposited by condensation of the metal vapour on the surface of the polymer precursor, by chemical vapour decomposition (e.g. Nickel carbonyl when heated to temperature above $120^{\circ} \mathrm{C}$ ) or by electro-deposition. Thus, the metallic foam takes the same structure as the original polymer. The polymer is then removed using thermal treatment leading to hollow ligaments with a thickness of a dozen micrometres of the coated cellular metals. Metals such as $\mathrm{Ni}, \mathrm{Cu}$, and alloys $\mathrm{Ni}-\mathrm{Cr}$ are commercially available using these processes. Generally, the produced foams have relatively high void volume fractions (92\% -97\%). Foams of large range of cell sizes (from $0.5 \mathrm{~mm}$ to $3 \mathrm{~mm}$ ) can be fabricated (Banhart, 2001). Foams produced by metallic deposition are characterised by their good uniformity, high degree of porosity and hollow struts (Davies and Zhen, 1983).

- Investment casting methods (Ashby, 2000; Banhart, 2001): The metal open foam is constructed by replicating a chosen polymer foam structure, which defines the density and morphology of the final product. Typical void volume fractions range from 80 to $97 \%$ and pore sizes from 1 to $5 \mathrm{~mm}$. The process consists, first, of filling with a slurry heat resistant material into a polymer foam precursor, e.g. polyurethane, to form a mould. The polymer template is then removed by thermal treatment leaving a negative image of the foam. A molten metal or a slurry powdered metal is then filled into the mould and later sintered. The casting can be achieved by gravity or by applying pressure to fill in the narrow cavities. Particular attention should be paid in order to achieve a complete filling of the thin struts and remove the mould material without damaging the structure. 
- Other techniques are also developed to create open cell foams but are not commercially available. Among them, coating of open porous polymer by dipping it into slurries containing powdered metal. Casting liquid metal around granules or hollow spheres of low density, which allows the control of pore size distribution. The 3D printing is also promising technique for manufacturing of various morphologies of foam with high precision.

Morphologies of metal open cell foam are best described by analysing the structure of the template precursor. Polymeric open cell foams are often used as precursors for metallic foams. They are produced as a result of polymerisation reaction accompanied by gas generation, which leads to the porous form of the material. For example, a polyurethane foam is formed by generating $\mathrm{CO}_{2}$ as a result of the reaction between isocyanate and water, or other gas as a result of vaporisation of volatile liquid (Volz, 1965). The gas forms nearly spherical bubbles in the material in the plastic state. The increasing of the number of bubbles together with their expansion leads to contact and then to form cellular structures. Generally, cells form dodecahedrons with pentagonal faces (Volz, 1965). The rupture of liquid films between two bubbles leads to interconnect cells. During the foaming process of the polyurethane foam template, several forces can modify the cells shape. Indeed, the manufacturing process involves various phenomena such as drainage (the transport of liquid through the foam, driven by pressure difference or gravity) and phases stabilisation, etc., which may lead to some unwanted changes in foam structures. A gradient in density, pore size and pore elongation may occur as consequence of gravitationally induced drainage during the manufacturing of the polyurethane foam used as a template (Simone and Gibson, 1998). The rupture of foam struts may occur due to surface adsorption or thinning. During foaming, surface tension pull material into the nodes, which leads to thicker Plateau's borders and thinner edges (Ip et al., 1999). It has been reported that adding particles into molten metal may help for phase stabilisation. In fact, particles increase the surface viscosity and hence retard drainage in films. Further discussion on the foam structure and Plateau's law is giving in the next sections.

\subsection{Open cell foam structure and Plateau's laws}

The characterisation of the open cell foam structure includes physical parameters such as the relative density $\rho_{r e l}$ and the pore density ppi (pore per inch) as well as the geometrical parameters such as the strut thickness, cell diameter $d_{c}$, node volume, and topological parameters. The influence of processing parameters on the structure of metal open cell foams has hardly been investigated in the literature. This is required for design optimisation and control of the foam structure in order to avoid defects. The influence of the casting and mould temperatures on the mechanical properties of investment-cast open-pore aluminium foams has been investigated by Fischer (Fischer et al., 2013). It was reported that decreasing mould temperature and increasing casting temperature lead to increased mechanical properties due to an increase in the strut diameter resulting from the mould filling ability of the melt. The template is required to be well ventilated, with relatively large pores diameter, and with thick struts which are uneasily deformable to preserve the same structure (Wang and Wang, 2001). Metallic foams prepared by using the investment cast or the deposition methods possess the advantages that the void fraction and pore structure can easily be controlled. Generally, the polymer foam precursor used for metal foams manufacturing leads to relative densities in the range of $2 \%$ to $80 \%$ and pore densities in the range of 10 ppi to 100 ppi (Matz, 2014).

Ashby (Ashby, 2000) has observed the structure of metal foam from different suppliers (Cymat, Mepura, Shinko) and has reported that the structures of open metal foams resemble those of soap films. It appears to be governed by the surface energy minimisation. This imposes the properties of the structure of soaps foams formulated by Joseph Plateau and grouped under the name of Plateau's law (Plateau, 1873). These laws define the network geometry of dry soap froth as 3D polyhedral cells separated by surfaces with constant mean curvature. Three curved faces meet at equal angles of $\sim 120^{\circ}$ and form an edge, and four edges meet at equal tetrahedral angles of $\sim 109^{\circ}$ at a vertex. The so-called Kelvin structure (formed of truncated octahedral) is an example of foam structure respecting the laws of Plateau, but Weaire (Weaire and Hutzler, 1999) has demonstrated that it is not the only possible shape allowing the minimisation of the surface energy. The foams observed by Ashby have polyhedral cells which also satisfy the surface energy minimisation. However, these foams are anisotropic with several faces (throats) appeared to have non-uniform curvature and some broken walls that still hang in place (Ashby, 2000).

\subsection{Structure analysis of foam using iMorph and construction of graphs}


iMorph (Vicente and Brun, 2009; Brun et al., 2008) is an open software developed to characterise porous media. The software provides macroscopic information such as porosity, anisotropy, cell orientations as well as geometrical information such as pore diameter, strut thickness or section, node volume and surface area, etc. It gives also topological description and generate graphs (networks of the solid and fluid phases).

The 3D construction of the numerical foam is performed by identifying, from grey level images obtained from $\mathrm{X}$-ray CT, the two phases of the sample (solid and air) by thresholding. At this stage of the analysis the porosity can be computed. The mesh of the solid surface, which can be used for CFD simulation, is obtained using marching cubes algorithm (Lorenson, 1987). An example of simulation of transport phenomena in open cell metallic foams, using mesh of the solid phase can be found in the work of Brun et al. (2009). Furthermore, the specific area can also be calculated from the interfacial meshes obtained.

The granulometry measurement gives information about the size distribution of pores using as a representative diameter that of the maximum included sphere. It is assumed that only one totally included ball is comprised into each pore. Segmentation of the porous phase is then made by applying the watershed transform to the pore distance map starting from the extracted markers (Meyer, 1991). A marker is the local extrema of the pore distance map and is attributed to each pore using the maximal included ball method (Beucher, 2005). The idea can be represented as flooding of different water sources at different regions and building barriers at the meeting points. Further information about image processing by iMorph can be found in the user guide (Vicente, 2017).

After segmentation and identification of the porous cells, the graph of the fluid phase can be constructed. This graph is formed by associating to each porous cell a vertex and to the connection between two neighbour cells an edge. Two porous cells are considered neighbours if they have a common border defined during the watershed process. These border areas are called windows or throats (see Fig.1). As discussed above, foams can have irregular structure, with elongated cells which complicate the segmentation and the construction of graphs. The incomplete cells at the boundary of the 3D numerically constructed foam can be included in the graph by considering a vertex as an approximated centre of the pore volume (Vicente, 2006). The graph of solid, intuitively, represents the solid matrix where vertices are solid nodes and edges are struts. Assuming that open cell foam structure satisfies Plateau's laws, the graph of the solid is deduced from the porous phase structure: Vertices are created at the meeting of three porous cells and edges at the meeting of four porous cells.

\section{Graphs and cell Method}

The modelling approach we present in this paper employs the topology of the foam structure for discretisation of the computational domain as a graph representation. A classical approach used in the literature for representing an equivalent homogeneous foam structure as graph, is through a circuit graph analogic to an electrical circuit (Calmidi et Mahajan, 1999; Bhattacharya et al., 2002). Here, we are interested in representing the real topology of heterogeneous foam structures (the solid and the fluid phases) as graphs, which also contain geometrical data. This topology preserving model leads to reduction of the computational time for the simulation as compared to the finite element meshing.

After introducing the particularity of the foam structure and the graph construction based on Plateau's laws, we discuss in this section the elements of the graph based on the graph theory. The graph theory is used in modelling of systems involving networks. The vertices of a graph can be considered as energy storage and the edges as a connection or transmission (transfer of energy). The relation between the elements of the graph are specified by incidence matrix. For modelling of physical systems, the cell method was used to construct discrete topological objects, oriented and associated with physical variables. We give a brief introduction to the Cell method and the discrete modelling of conservation laws on Cell-complex. 


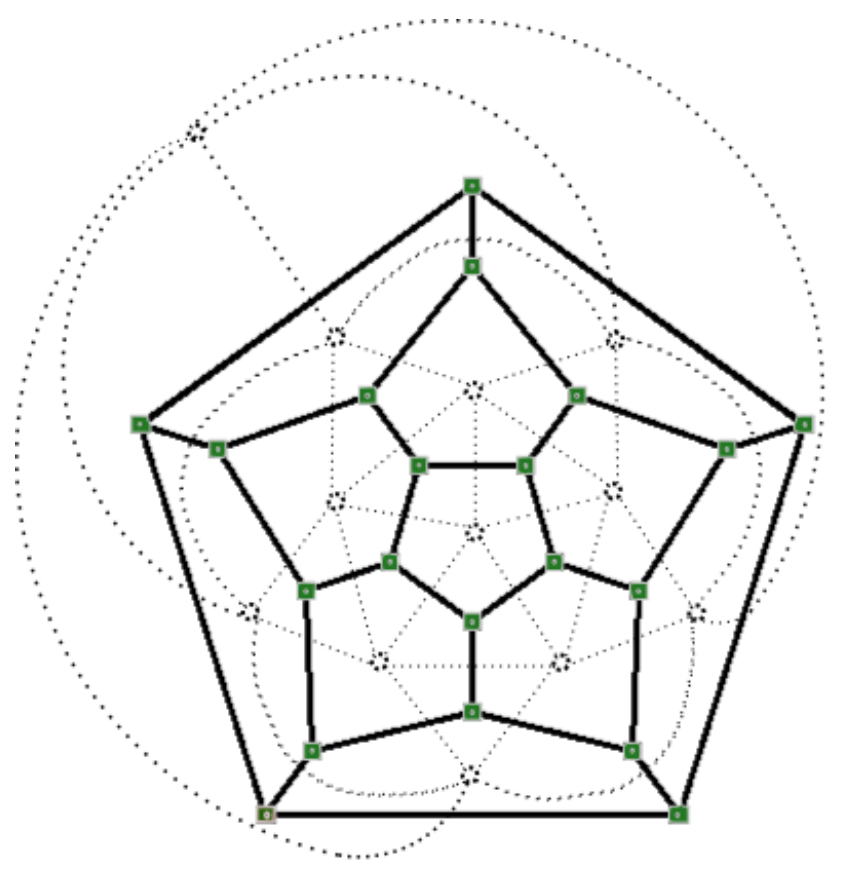

Fig.2 Schematic representation of the graph $\mathrm{H}$ of an Icosian game (continued line) and it is dual graph H' (dotted line).

\subsection{Brief introduction to the graph and cell theory}

We give, here, some definitions of the graph and its elements. The dodecahedron graph $\mathrm{H}$ of the Icosian game will be used as an example for illustration (Fig.2). A graph $\mathrm{H}$ is defined by a finite nonempty set of points called vertices and denoted by $\mathrm{V}$. Vertices are connected by a set of lines called edges and denoted by E. Two vertices of an edge are said to be incident with the edge and viceversa. Two edges are said to be adjacent if they are incident with the same vertex, and two vertices of an edge are said to be adjacent. This leads us to define matrices which define the relation between the elements of the graph.

- The incidence matrix $M$ is a $N_{V} \times N_{E}$ matrix, where $N_{V}$ is the number of vertices and $N_{E}$ is the number of edges.

- The adjacency matrix $A$ is a $N_{V} x N_{V}$ matrix, which gives the number of edges joining $V_{i}$ and $V_{j}$.

- The degree matrix $D$ is a $N_{V} \times N_{V}$ diagonal matrix, which gives information about the number of edges connected to each vertex.

The adjacency matrix and the degree matrix allow to construct the Laplacian matrix of a graph $\mathrm{L}=\mathrm{D}-\mathrm{A}$.

\subsection{Cell method}

The cell method is a computational approach to solve field equations from algebraic formulation, thus avoiding the discretisation of the differential equations (Pani and Taddei, 2013). This is based on the fact that the physical variables are referred to geometrical elements such as point, line, surface and volume.

Table.1 Cell method, analogy of geometrical elements with Cell-complex and possible associated physical variables

\begin{tabular}{|c|c|c|c|c|}
\hline $\begin{array}{l}\text { Geometrical } \\
\text { elements }\end{array}$ & $\downarrow$ & & & 5 \\
\hline Graph elements & Vertex & Edge & Face & Volume \\
\hline Cell complex & 0 -cell & 1-cell & 2-cell & 3-cell \\
\hline Associated variables & Temperature & Driving force & Heat transfer rate & Internal energy \\
\hline
\end{tabular}




\section{Cell-complex}

Cell-complex, denoted by K, founded by Poincaré in 1895 (Poincaré, 1895), defines a graph as a set of cells (discrete spacial elements) of various dimensions. In the three dimensional space, cells can be vertices, edges, faces and volume denoted as 0 -cell, 1-cell, 2-cell and 3-cell respectively (see Table.1). The maximum number $\mathrm{p}$ of cells is one more dimension $(n+1)$ of the corresponding space $R^{n}$. The connections between $(p-1)$-cell and $p$-cell are defined using incidence matrices. The incidence numbers have value of -1 or 1 if the $(p-1)$-cell and $p$ cell are mutually incident and 0 if they are not. In three-dimensional space, we define three incidence matrices: $\mathrm{M}_{1}$ (0-cell x 1-cell), $\mathrm{M}_{2}$ (1-cell x 2-cell) and $\mathrm{M}_{3}$ (2-cell x 3-cell). $\mathrm{M}_{1}, \mathrm{M}_{2}$ and $\mathrm{M}_{3}$ are the discrete version of the operators 'grad', 'rot' and 'div' respectively.

\section{Dual Cell-complex}

Sometimes, it is more convenient to use a dual representation of the Cell-complex since it allows for coupling two physical phenomena (e.g. electric and magnetic fields) or interacting phases (e.g. solid and fluid in porous material). The dual of K-complex is constructed as follow: to each p-cell of the K-complex, a dual ( $\mathrm{n}-\mathrm{p}$ )-cell is associated, to define a dual complex denoted by $\widetilde{\mathrm{K}}$ and viceversa (Tonti, 1976). In other word, if we consider a point inside each 3-cells of $\mathrm{K}$, another Cell complex can be constructed by taking these points as vertices, which is called dual complex and denoted by $\widetilde{\mathrm{K}}$. To give it physical meaning the vertex associated to the dual $3-$ cell can be considered as the centre of gravity of the object. For illustration, the dual of the dodecahedron in two-dimensional space in Fig.2 is drawn in dotted line. It can be observed that for each 2-cell, 1-cell and 0-cell of $\mathrm{K}$ correspond a 0 -cell, 1-cell and 2-cell of $\widetilde{\mathrm{K}}$. The exterior region of $\mathrm{K}$ is considered as a 2-cell and a corresponding 0 -cell of $\widetilde{\mathrm{K}}$ was included in the graph.

\section{Orientation of cells}

Cells are conventionally oriented in order to define signs of physical variables associated to the objects. For example, a vertex has a negative orientation if it is conceived as a source and a positive orientation if it is conceived as a sink. This orientation is intrinsic meaning it lies on the element itself, thus called inner orientation. On the contrary, an outer orientation of a vertex is obtained from the inner orientation of the volume containing the vertex (represented by a sense of rotation around the segments in 3D space). Illustrations of inner and outer orientations of p-cells can be found in these two references (Tonti, 1976; Tonti, 2001). Since a vertex of K-complex is associated with a volume of the dual $\widetilde{\mathrm{K}}$-complex, it can be generalised saying that the outer orientation of p-cell is the orientation of its dual $(n-p)$-cell (Veblen and Whitehead, 1932). The outer orientation of a 3-cell is represented by the outward or the inward normal, while the outer orientation of point is defined fixing a sense of rotation around the lines that arrive or leave the vertex. The orientations of p-cells are taken into consideration through the sign of the incidence numbers of the incidence matrices.

Practically, in order to orient a p-cell, we need to consider p-simplex, which is the simplest geometrical object of $p$ dimensions (e.g., a triangle is the simplex in 2 dimensions and a tetrahedron is the simplex in 3 dimensions). An example of orientation of a tetrahedron is given in Annex.1. The idea is to orient a simplex and in an intuitive way it implies the orientation of its boundary (Franz, 1968). Orientation of simplexes can also be induced based on Möbius law of edges which states that orientation of two adjacent simplexes is such that the orientations induced on their common elements are opposite (Klein 1948).

\section{$\underline{\text { Chains }}$}

Physical variables can be assigned to each oriented p-cell (with inner or outer orientation) of a cell complex and assembled to define a chain. Consider, for example, a heat flux associated with every oriented 2-cell of a cell complex in the three dimensional space, the distribution of the heat fluxes in the considered region is described by the 2-chain : $\left\{\hat{\phi}_{1}, \hat{\phi}_{2}, \ldots \hat{\phi}_{n}\right\}$. The coefficients of these chains are the amounts of the physical quantity associated with every cell. If the orientation of the cell is changed, the coefficient of the chain undergoes a sign change. Moreover, the p-chains are algebraic entities, where those of the same dimensions can be added (Lefschetz, 1965). The boundary of the p-chain is defined as the (p-1)-chain and the process to form the boundary operator can be consulted in this reference (Tonti, 2013). 


\subsection{Conservation laws on cell complex}

The cell complex approach for modelling is analogous to finite modelling when a small volume element is considered and supposed to be in an equilibrium state. It may also be viewed as the representation of a set of discrete balance equations (Kotyczka and Maschke, 2017) written on the cells of K-complex. However, contrary to the cell complexes obtained by meshing a homogenous spatial domain, for a heterogeneous medium, the discretisation is made based on the geometrical elements of the material networks equivalent to discrete element method. For the model of open cell foam, the discrete elements are the node volumes of the solid network and the pore volumes of the fluid phase network. The transfer phenomena within the cells of a phase are expressed using the incidence matrices between the 3-cells and the 2-cells associated with the fluxes. Between the two phases, an additional incidence relation shall be used. In the sequel, we shall briefly recall the balance equations associated with the heat transfer in the open cell foam in the continuous and the discrete formulations.

Consider first the partial differential equation of the thermal balance of a continuous non-reactive phase:

$$
\rho C_{p} \frac{\partial T}{\partial t}=-\operatorname{div} \phi, \quad(\underline{\mathrm{Eq} .1})
$$

where $\mathrm{T}$ is a function of the three spatial variables $(\mathrm{x}, \mathrm{y}, \mathrm{z})$ and the time variable $t$, and $\phi$ is the heat flux. $\rho$ is the body density and $C_{p}$ is the heat capacity. Considering the heat is only flowed by conduction, the heat flux is expressed based on the continuum concept according to Fourier's law as

$$
\phi=-\lambda \operatorname{grad} T . \quad(\text { Eq.2) }
$$

where $\lambda$ is the thermal conductivity. For heterogeneous material such as open cell foams, the thermal conductivity varies within the medium. Thus, an average value of $\lambda$ over a temperature difference in one phase could be considered. In the literature, an effective thermal conductivity $\lambda_{\text {eff }}=f\left(\lambda_{s}, \lambda_{f}, \varepsilon\right)$ was considered for modelling of heat transfer in open cell foams as homogeneous media. Here, $\lambda_{s}$ is used for the solid phase and $\lambda_{f}$ for the fluid phase.

Now, we write the set of discrete balance equation for every control volume $v_{i}$ associated with a node $n_{i}$ and with boundary faces $\partial v_{i j}$

$$
\frac{d}{d t} \int_{v_{i}} u_{i}^{s} d v=-\sum \int_{\partial v i j} \phi_{i j}^{s} d s,(\underline{E q .3})
$$

$u_{i}^{S}$ is the internal energy density for the control volume $v_{i}$ associated with a solid node $n_{i}$ and $\phi_{i j}$ is the heat flux is expressed as a function of a driving force associated with the edge $e_{i j}$ (Fig.3-left) as

$$
\phi_{i j}^{s}=-\lambda_{s} F_{i j} \quad, F_{i j}=T_{j}-T_{i} .(\underline{E q} .4)
$$

The set of balance equations has the structure of a finite-dimensional dissipative port Hamiltonian system, which retains both the definition of the balance equations and driving forces and accounts for the topology of the open cell foam using the co-incidence matrices (Kotyczka and Maschke, 2017; Scheuermann et al., 2019).

For the heat transport into the two phases (solid and fluid) (Fig.3-right), two coupled discrete balance equations are written as:

$$
\begin{aligned}
& \frac{d}{d t} \int_{v_{i}} u_{i}^{s} d v=-\sum \int_{\partial v i j} \phi_{i j}^{s} d s-\sum \int_{\partial V i k} \phi_{i k}^{f s} d s,(\underline{\mathrm{Eq} .5}) \\
& \frac{d}{d t} \int_{v_{k}} u_{k}^{f} d v=-\sum \int_{\partial v k l} \phi_{i j}^{f} d s-\sum \int_{\partial v i k} \phi_{i k}^{f s} d s .(\underline{\text { Eq.6) }})
\end{aligned}
$$

Fourier's law is also used for the fluid phase; the heat flux is expressed as a function of a driving force associated with the temperature difference between two adjacent vertices of porous cells

$$
\phi_{k l}^{f}=-\lambda_{f} F_{k l} \quad, F_{k l}=T_{l}-T_{k} \text { (Eq.7). }
$$

Near to the node surface, in the boundary layer at the interface fluid - solid, the heat transfer between the two phases is expressed according to Newton's law of cooling as:

$$
\phi_{i k}^{s f}=-h A_{i k} F_{i k} \quad, F_{i k}=T_{i}-T_{k} \quad(\underline{\text { Eq.8}})
$$


where $h$ is the heat transfer coefficient between the solid surface and the surrounding fluid and $A_{i k}$ is the heat transfer surface area.

In this section, the heat transport is limited to the conduction into the two phases but this approach can be extended to take into account the convection by involving the motion of the fluid, and the radiation. From the above equations, we can see that besides the topological information of the fluid and the solid phases, geometrical information such as the volume of node and the surface areas are also necessary for modelling of heat transfer.
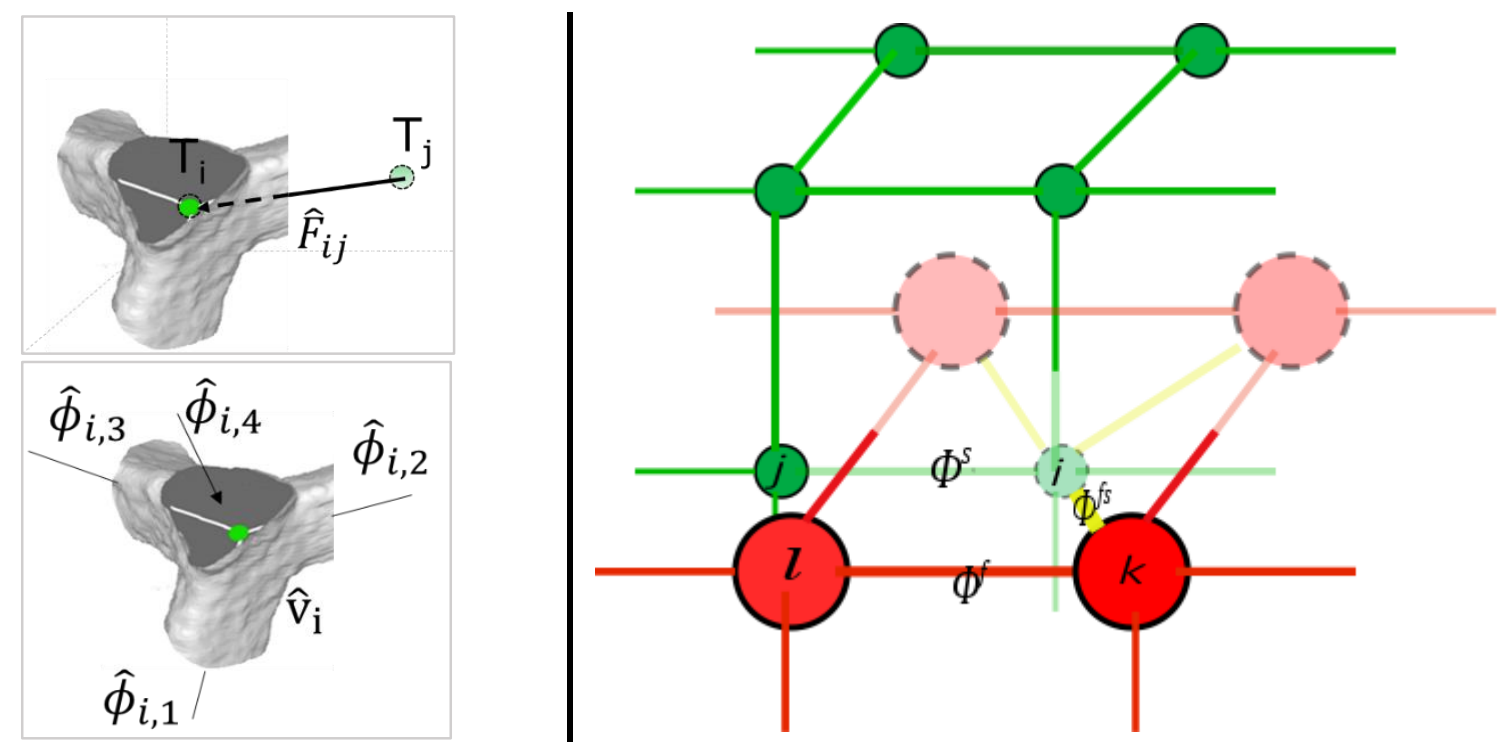

Fig.3 (left) Schematic representation of driving force associated with an edge (up) and heat transport over a controlled volume (down). (right) Regular structure scheme of the transfer between two phases solid - fluid.

\section{Graph construction using X-ray CT and iMorph}

In this section, we present the construction of graphs based on two samples of open cell foams. The extraction of geometrical and morphological information from different kind of cellular materials using iMorph can be consulted in these references (Vicente et al., 2006; Brun et al., 2007; Brun et al., 2008; Mollicone et al., 2014; Kumar et al., 2014). The modelling approach presented in this paper has led to extra implementation in the software iMorph for the construction of the solid and fluid phase graphs and extraction of specific geometrical measurements. The graph construction based on Plateau's law is discussed here. However, iMorph allows generating other graphs based on the skeleton of the porous material for example. The raison for choosing the Plateau's graph in this work is to take advantage of the duality of the graph of solid and fluid for modelling of the interphase phenomena.

\subsection{Materials and methods}

Samples: two samples have been chosen to be studied in this paper:

(i) Kelvin's tetrakaidecahedron cell (Fig.4-left) (Thomson, 1887), with regular structure, has 14 flat faces (6 quadrilaterals and 8 hexagonal faces), 36 straight edges and 24 vertices. The sections of the foam struts are convex triangles. This shape provides a minimum surface area to volume ratio due to the foam manufacturing process, which requires a minimum surface energy state for the bubble to attain equilibrium and the process to reach steady-state (Krichnan, 2006). Kraynik (Kraynik, 2003) has reported that the structure of Kelvin cell satisfies Plateau's law, which is suitable for our graph construction approach. 
Polyhedral open cell foam (Fig.4-right) has an irregular structure with cells having pentagonal and hexagonal faces (about 10 to 14) (Calmidi and Mahajan, 1999). The struts are thin but not perfectly straight and the Plateau's borders are observed at the strut cross section (convex triangular). We assume that the foam structure follows the Plateau's law in order to generate the graph of solid phase.

The Kelvin cell is manufactured by direct casting method and the polyhedral open cell foam by the investment casting methods. The Table. 2 gives information about the two samples, including dimensions and properties provided from suppliers. The Table. 3 gives further geometrical and morphological information obtained from iMorph analysis.
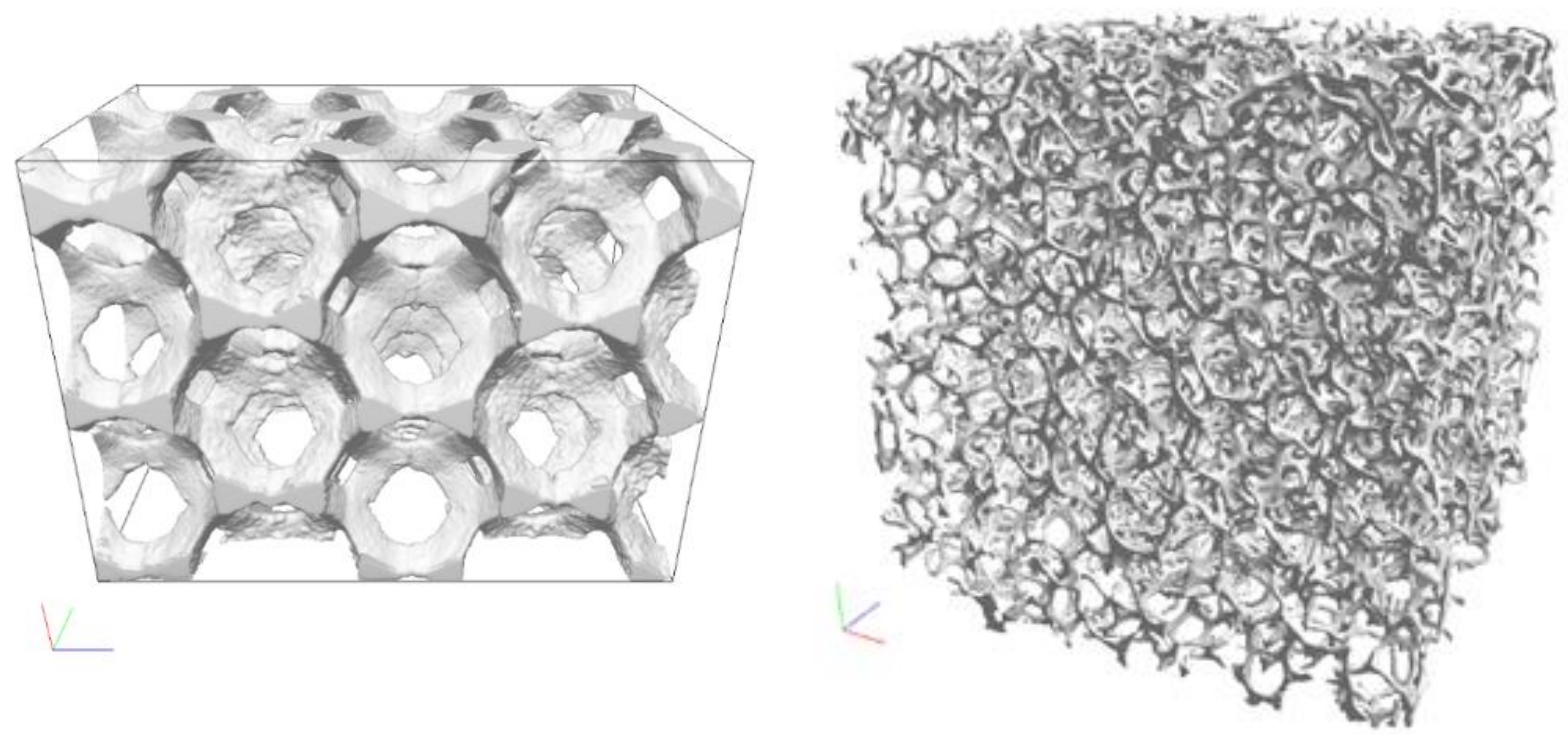

Fig.4 3D construction of Kelvin cell (left) and polyhedral cell foam (right) obtained foam X-ray CT scans and using iMorph for image processing.

Table.2 Information about the open cell foam samples.

\begin{tabular}{lll}
\hline & Kelvin cell foam & Polyhedral cell foam \\
\hline Supplier & Alveotec & Fraunhofer \\
Dimensions & $32 \times 27 \times 42 \mathrm{~mm}$ & $40 \times 40 \times 40 \mathrm{~mm}$ \\
Porosity & $\sim 0.8$ & $0.93-0.96$ \\
Pore density & $\sim 2 \mathrm{ppi}$ & $10 \mathrm{ppi}$ \\
\hline
\end{tabular}

\section{X-ray Computed Tomography}

$\mathrm{X}$-ray Computed Tomography is a non-destructive technique for analysis of solid bodies, where images of planes (Slices) can be assembled to form a 3D image (ASTM E1441-00, 1992). When the X-rays go through the sample to be analysed, they are attenuated differently depending on the density and the atomic number of phases (solid, gas, etc.) and recorded to form images with different grey level pixels. The X-ray tomography was performed on vtome $x$ apparatus provided by Phoenix X-ray company. The minimal pixel size is $2 \mu \mathrm{m}$ and the resolution is defined relative to the size of the samples. Here, the resolution of the X-ray scans is $40 \mu \mathrm{m}$ for the two samples.

\section{iMorph software}

Prior to the 3D construction of the foam samples, the images may need to be processed using some available options in the software e.g., to fill the pore cavities enclosed into the solid nodes and struts or in the other way 
around to eliminate some suspended small volume in the porous phase due to image resolutions. These may affect the construction of the graphs using iMorph.

Table.3 gives some geometrical measurements performed on the Kelvin cell and the polyhedral cell foams. We define the pore diameter, denoted $D_{p}$, as the diameter of a sphere of equivalent pore volume and the node diameter, denoted $D_{n}$, as the diameter of a sphere of equivalent node volume. Other geometrical measurements such as the diameter of the largest included sphere can also be obtained from iMorph. The surface area of a node, $S_{n}$, is extracted by using the mesh of the solid phase and counting voxels at the solid-fluid interface for each solid node and the strut section, $S_{s}$, is obtained from voxels at the junction between two nodes. Moreover, information about the porous cell shape and orientation can be obtained by investigating the 3D inertia matrix of the equivalent ellipsoid of the porous cells.

In the case of hollow struts (for foams manufactured by metal deposition for example), two porosities can be computed: the total porosity, which includes voxels of hollow struts in the computation of the porous phase volume and the open porosity, which excludes voxels of the hollow struts. Ruptures of struts are, sometimes, created as results of the segmentation when the wall of the struts is very thin or/and the tomography resolution is not high enough. This can be fixed by using some image processing options in iMorph.

Table.3 Average geometrical properties of the two samples obtained using iMorph.

\begin{tabular}{l|cccccc}
\hline Samples & Total porosity & $\begin{array}{c}\text { Specific area } \\
\left(\mathrm{m}^{2} / \mathrm{m}^{3}\right)\end{array}$ & $\begin{array}{c}D_{p} \\
(\mathrm{~mm})\end{array}$ & $\begin{array}{c}D_{n} \\
(\mathrm{~mm})\end{array}$ & $\begin{array}{c}S_{n} \\
\left(\mathrm{~mm}^{2}\right)\end{array}$ & $\begin{array}{c}S_{s} \\
\left(\mathrm{~mm}^{2}\right)\end{array}$ \\
\hline $\begin{array}{l}\text { Kelvin cell } \\
\text { foam }\end{array}$ & 0.81 & 306.2 & 13.1 & 4.2 & 60.4 & 1.1 \\
\hline $\begin{array}{l}\text { Polyhedral cell } \\
\text { foam }\end{array}$ & 0.94 & 332.4 & 5.9 & 1.2 & 5.2 & 0.23 \\
\hline
\end{tabular}

\subsection{The graphs of solid and fluid phases and the coupling graph}

iMorph allows to construct two 3D graphs, called the primal representative graphs, of the solid and fluid phases. The Fig.6 shows these graphs (fluid phase on the left and solid phase on the right), where each graph is composed of 0-cells and 1-cells. For the fluid phase, the 0-cell is a vertex associated to each pore and the 1-cell is associated to each edge connecting two adjacent pores vertices. For the solid phase, the 0 -cell is, intuitively, associated to a solid node and the 1-cell to a solid strut.

The dual representative graph for each phase is constructed as follow: for the fluid phase, the 3-cells are associated to the pore volumes and the 2-cells are associated to the throat surfaces of the porous cells (see Fig.1). In the same way, for the solid phase, the 3-cells are associated to the volume of solid nodes (4 associated half struts) and the 2-cells are associated to the interface sections (junctions) between adjacent nodes.

The primal and the dual representative graphs of each phase constitute the representative graph (structure representation), which is the 3-cell complex presented in section 3.2. The dual representative graph is precisely the dual of the K-complex given by the primal representative graph. The primal 0-cells are located inside the dual 3-cells and the primal 1-cells cross the dual 2-cells. Besides, the number of the primal 0-cells is equal to the number of the dual 3-cells and the number of primal 1-cells is equal to the number of the dual 2-cells.

With each p-cell of the K-complexes, the physical variables are associated to the corresponding chains and cochains (the dual of the chain) over the two graphs. That amounts to associate, for each phase a temperature to a primal 0-cell, a driving force to a primal 1-cell, a heat flux to a dual 2-cell and an internal energy to a dual 3cell. The incidence matrix (2-cell x 3-cell) of the dual representative graphs allows computing internal energy from the heat fluxes on the boundary surfaces as giving by the balance equation Eq.3. The transpose of this matrix is the co-incidence matrix (1-cell x 0 -cell) of the primal representative graphs, which allows the computation of the driving forces from the temperature differences as shown in Eq.4.

In order to compute the heat transfer between the two phases solid-fluid, another 3D graph called the coupling graph is defined. This graph is obtained by considering a tetrahedral unit volume around every solid node, where the summits are the vertices or 0 -cells of the fluid phase primal representative graph (see Fig.5). This particular geometry (tetrahedron) comes from the construction of the solid graph using Plateau's laws. Indeed, since the nodes of the solid are defined at the meeting point of the four porous cells around it, the centres of 
these pores delimit a tetrahedron. The heat flux between the two phases is integrated over the 2-cell associated with the solid-fluid interfaces of the coupling graph (blue area in Fig.5). Using the dual representation, a driving force is associated to 1-cells (dual to the 2-cells) relating a solid vertex and an adjacent pore vertex (one of the tetrahedral summit). Again, the chains define the temperature associated with the nodes and the edges associated to the driving forces can results as temperature difference between the two 0-cells

This approach is also used to construct the representative graphs and the coupling graph for the polyhedral open cell foam assuming that the solid geometry obeys the Plateau's laws (see Fig. 7). It is obviously more complicated to construct the graph of the irregular foam structure because of manufacturing defects such as elongated porous cells and broken struts. A post-processing of the graph constructed by iMorph is needed for checking.

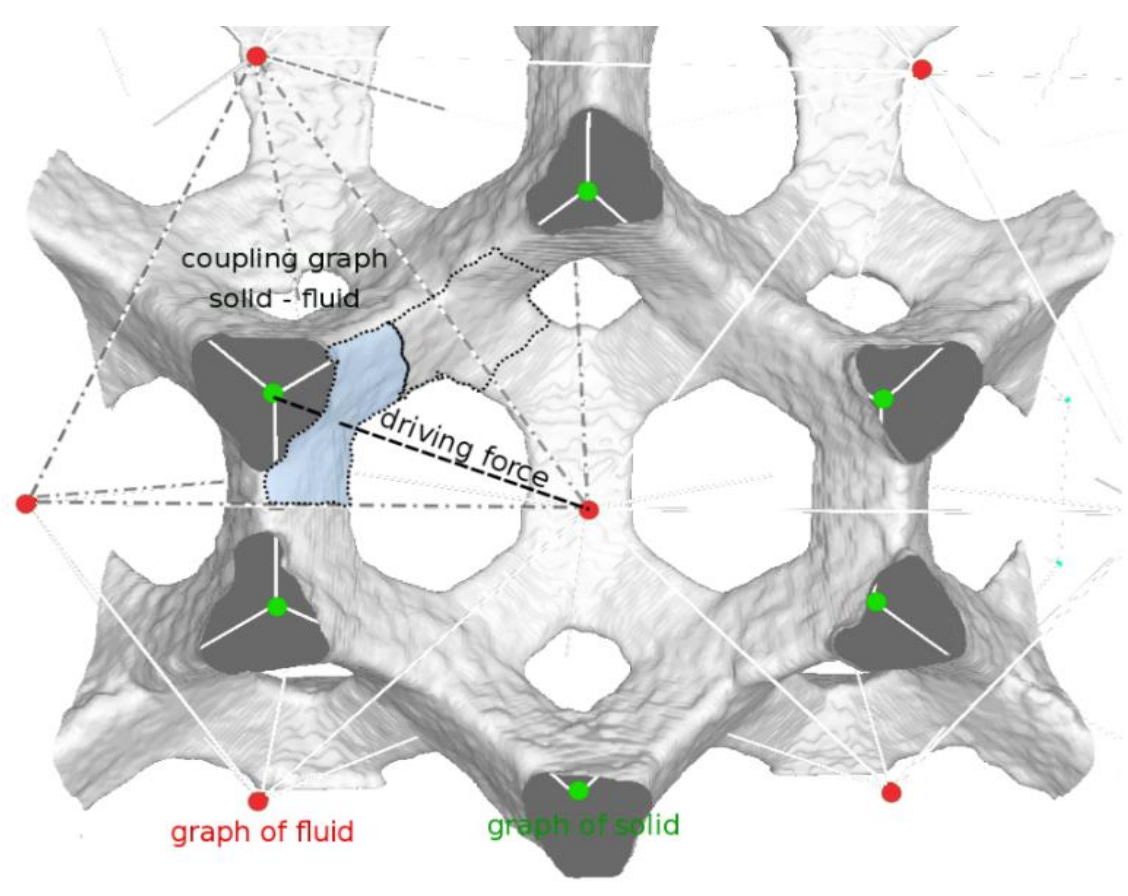

Fig.5. Illustration of the three graphs constructed from a Kelvin cell foam : the solid and fluid primal representative graphs and the coupling graph.

\subsection{Extended graph - port variables}

The graph generated by iMorph can be defined to be not extended and then corresponds to an isolated system or to be extended meaning that it allows for open systems including to boundaries. In this sub-section, we are going to discuss the difference between the two types of graphs and how it can be used for modelling.

Fig. 6 shows the primal representative graphs of the Kelvin cell foam: the fluid graph where vertices are represented by red dots and the solid graph where vertices are green dots. The extended graphs $\mathbf{C}$ and $\mathbf{D}$ of each phase are placed beneath the not extended $\mathbf{A}$ and $\mathbf{B}$. It can be observed that more vertices appear in the extended case which are attributed to the boundaries defined by the cuboid domain. In the not extended graph of the fluid phase, a vertex is attributed to each incomplete porous cell at the borders. As the graph of the solid phase is obtained based on the Plateau's laws (at the meeting of the porous cells), some vertices and edges of the solid graph at the boundaries may not be defined in the not extended graph. This is a limitation of the graph construction based on the Plateau's laws.

The extended fluid graph adds extra vertices attributed to the boundary incomplete porous cells, called boundary vertices $V_{b}$. The latter are connected by edges to the internal vertices and by boundary edges $E_{b}$ to the cuboid domain faces (see Fig.6C). The set of $E_{b}$ permits the interconnection with other graphs or with the environment. The extension of the graph of fluid phase, consequently, induces the extension of the graph of solid phase (using 
the Plateau's laws at the boundary incomplete porous cells) (see Fig.6D). Moreover, the dual representative graphs and the coupling graph are also extended.

The extended graphs are particularly interesting for modelling of the transport phenomena in open systems since the subset of $V_{b}$ can serve to define port variables that are the interface variables of the transport phenomena with the environment of the foam. A driving force can be attributed to the edges connecting the boundary vertices and the ones inside and the heat fluxes to the boundary faces. The boundary vertices allow for interconnection with other extended graphs (e.g. application for catalytic reactor with packed foams). For control purposes, it can be used to define the input and output variables (van der Schaft, 2013).
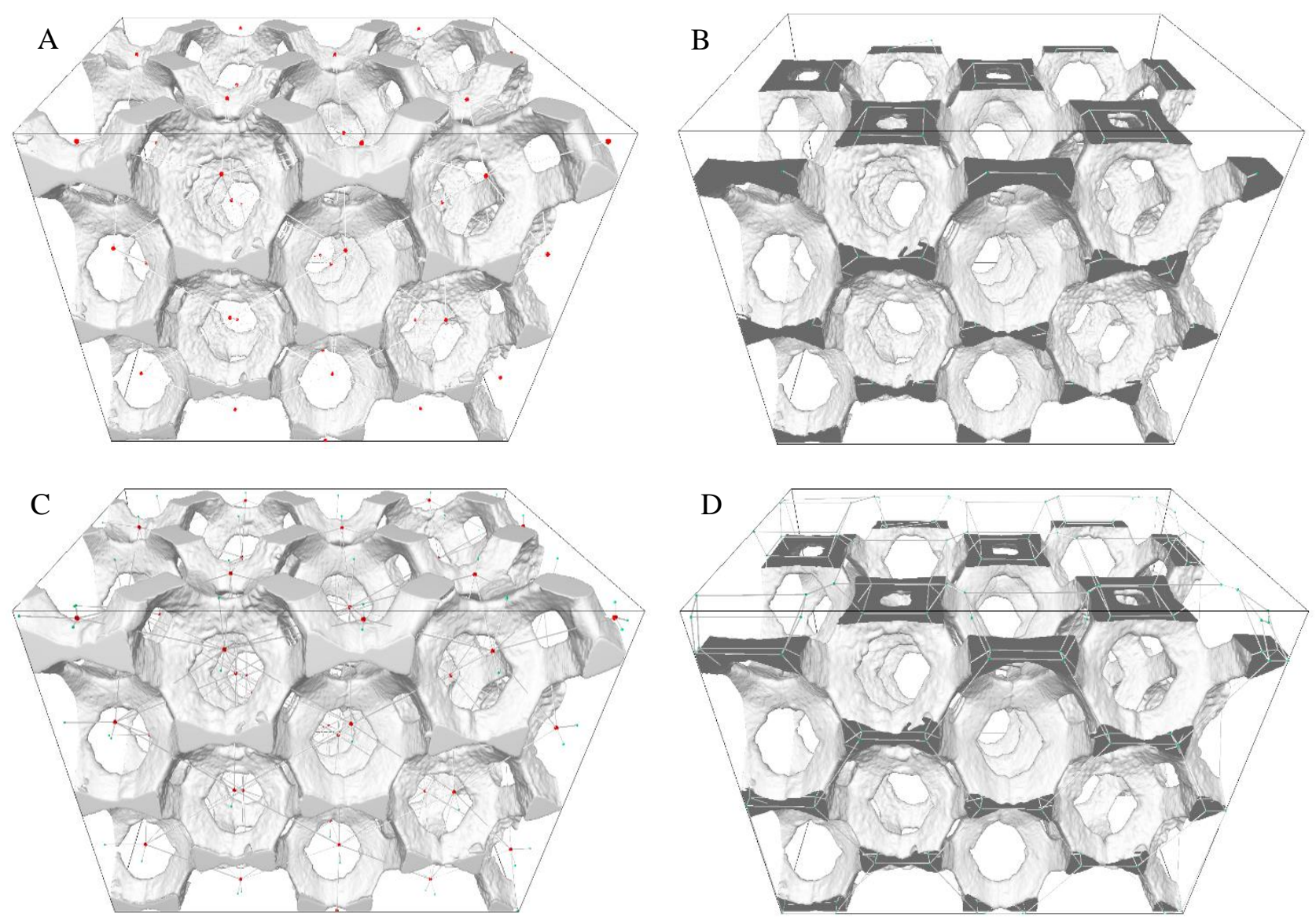

Fig.6 (up) "Not Extended" graphs of Kelvin cell foam: A is the fluid phase graph and $\mathbf{B}$ is the solid phase graph. $\mathbf{C}$ is the "Extended" graph of $\mathbf{A}$ and $\mathbf{D}$ is the "Extended" graph of $\mathbf{B}$. 


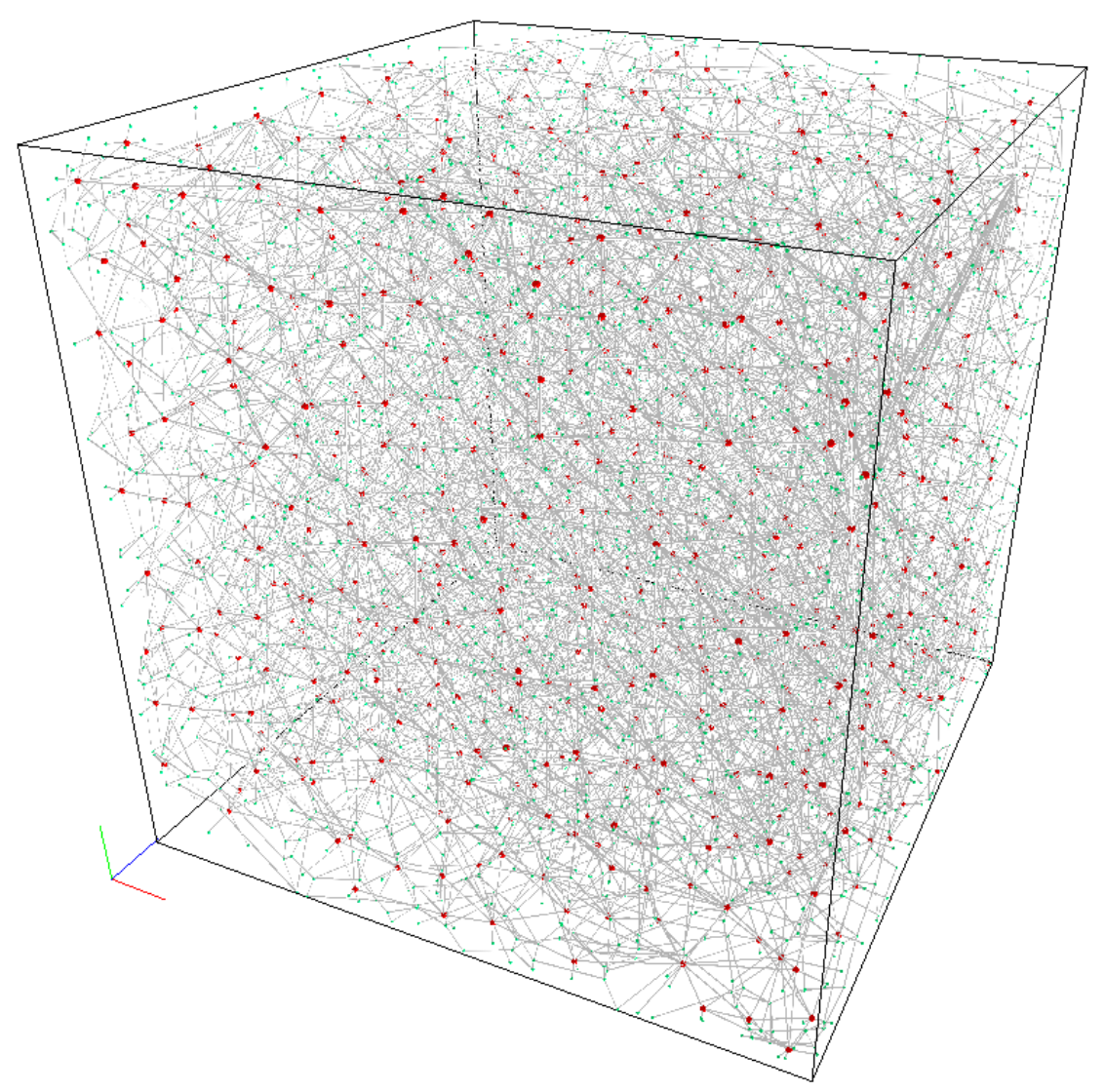

Fig.7 The primal representative graphs (solid phase with green vertices and fluid phase with red vertices) of irregular polyhedral open cell foam obtained from iMorph.

\section{Conclusions}

In this paper, the structure of open cell foams is studied as an interesting case to generate graphs. The particularity of the open cell foams is that the graph can be observable from the network of the solid matrix where vertices are nodes and edges are struts. The use of graphs for modelling of transport phenomena offers a simplification and model reduction in comparison to modelling based on meshing of the whole domain (e.g. finite element method). Thus, it gives a possibility for modelling of large size cell foams with large networks as shown in Fig.7. Besides, the use of the duality of the graphs gives a starting point for coupling physical phenomena or interphase interactions. The interconnection of the different elements (p-cell) of the graphs (vertex, edge, face and volume) are mathematically written using incidence matrices. The latter is combined with energy balances and driving forces in Port-Hamiltonian System formulation (Kotyczka and Maschke, 2017; Scheuermann et al., 2019). Finally, the graph based modelling is not restricted to open cell foams structure but can be used for different porous structures.

\section{References}

Amani Y., Takahashi A., Chantrenne P., Maruyama S., Dancette S., Maire E. (2018). Thermal conductivity of highly porous metal foams: Experimental and image based finite element analysis. Int. J. Heat Mass Tran.,122, pp. 1-10.

Ashby M.F., Evans A., Fleck N.A., Gibson L.J., Hutchinson J.W., Wadley H.N.G. (2000). Metal foams: a design guide. Massachusetts: Butterworth-Heinemann, 251.

ASTM: E 1441-00 Standard guide for computed tomography (CT) Imaging. ASTM (2000).

Banhart J. (2001). Manufacture, characterisation and application of cellular metals and metal foams. Prog. Mater. Sci., 46, pp. 559-632.

Banhart J. (2006). Metal foams: production and stability. Adv. Eng. Mater., 8, pp. 781-794.

Beucher S., (2005) Numerical residues. In Mathematical Morphology: 40 Years on. Paris, pp. 23-32. 
Bhattacharya A., Calmidi V.V., Mahajan R.L. (2002). Thermo-physical properties of high porosity metal foams. Int. J. Heat Mass Tran., 45, pp. 1017-1031.

Boomsma K., Poulikakos D. (2001). On the effective thermal conductivity of a three dimensionally structured fluidsaturated metal foam. Int. J. Heat Mass Tran., 44, pp. 827-836.

Bracconi M., Ambrosetti M., Maestri M., Groppi G., Tronconi E. (2018). A fundamental analysis of the influence of the geometrical properties on the effective thermal conductivity of open-cell foams. Chem. Eng. Process. -Process Intensification, 129, pp. 181-189.

Brun E., Vicente J., Topin F., Occelli R., Clifton M.J. (2009). Microstructure and transport properties of cellular materials: Representative volume element. Adv. Eng. Mater., 11, pp. 805-810.

Brun E., Vicente J., Topin F., Occelli R. (2007). From 3D imaging of structures to diffusive properties of anisotropic cellular materials. In: Continuous models and discrete systems 11, Paris, pp. 121-127. 〈hal-00199430〉.

Brun E., Vicente J., Topin F., Occelli R. (2008). iMorph: 3D morphological tool to fully analyse all kind of cellular materials. Cellmet'08, Dresden, Germany.

Calmidi V.V., Mahajan R.L. (1999). The effective thermal conductivity of high porosity fibrous metal foams. J. Heat Transfer, 121, pp. 466-471.

Davies G.J., Zhen S. (1983). Review Metallic foams: their production, properties and applications, J. Mater Sci., 18, pp. 1899-1911.

De Jaeger P., T'Joen C., Huisseune H., Ameel B., De Paepe M. (2011). An experimentally validated and parameterized periodic unit-cell reconstruction of open-cell foams, J. Appl. Phys., 109, pp. 103519.

Fischer S.F., Schüler P., Fleck C., Bührig-Polaczek A. (2013). Influence of the casting and mould temperatures on the (micro) structure and compression behaviour of investment-cast open-pore aluminium foams. ACTA Mater., 61, pp. 51525161.

Franz W. (1968). Algebraic topology. New York, F. Ungar Pub. Co., p. 170.

Frey M., Romero T., Roger A.C., Edouard D. (2016). Open cell foam catalysts for CO2 methanation: Presentation of coating procedures and in situ exothermicity reaction study by infrared thermography. Catal. Today, 273, pp. 83-90.

Giani L., Groppi G., Tronconi E. (2005). Mass-transfer characterisation of metallic foams as supports for structured catalysts. Ind. Eng. Chem. Res., 44, pp. 4993-5002.

Han X.H., Wang Q., Park Y.G., T'Joen C., Sommers A., Jacobi A. (2012). A review of metal foam and metal matrix composites for heat exchangers and heat sinks. Heat Transfer Eng., 33, pp. 1-22.

Ip S.W., Wang Y., Toguri J.M., (1999). Aluminium foam stabilisation by solid particles. Can. Met. Quart., 38, pp. 81-92.

Jang W-Y., Kraynik A.M., Kyriakides S. (2008). On the microstructure of open-cell foams and its effect on elastic properties. Int. J. Solids Struct., 45, pp. 1845-1875.

Klein F. (1948). Elementary mathematics from an advanced standpoint. Geometry, 39, pp. 201-136.

Kotyczka P., Maschke B. (2017). Discrete Port-Hamiltonian formulation and numerical approximation for systems of two conservation laws. Automatisierungstechnik, 65, pp. 308-322.

Kraynik A.M. (2003). Foam structure: from soap forth to solid foams. Mater. Res. Soc., pp. 275-278.

Krishnan S., Murthy J.Y., Garimella S.V. (2006). Direct Simulation of transport in open cell metal foams. J. Heat Transfer, 128, 793-799.

Kumar P., Topin F., Vicente F. (2014) Determination of effective thermal conductivity from geometrical properties: application to open cell foams. Int. J. Therm. Sci., 81, pp. 13-28.

Lefebvre L-P., Banhart J., Dunand D.C. (2008) Porous metals and metallic foams: Current status and recent developments. Adv. Eng. Mat., 10, pp. 775-787.

Lefschetz S. (1965). Topology, $2^{\text {nd }}$ edn. Chelsea, New York

Liu P.S., Li T.F., Fu C. (1999). Relationship between electrical resistivity and porosity for porous metals. Mater. Sci. Eng. A, 268, pp. 208-215.

Lorensen W.E., Cline H.E. (1987) Marching cubes: A high resolution 3D surface construction algorithm. Computer Graphics, 21, pp. 163-169.

Mollicone J., Ansart F., Lenormand P., Duployer B., Tenailleau C., Vicente J., (2014) Characterisation and functionalization by sol-gel route of SiC foams. J. Eur. Ceram. Soc., 34, pp.163-169.

Matz A.M., Mocker B.S., Müller D.W., Jost N., Eggeler G. (2014). Mesostructural design and manufacturing of open-pore metal foams by investment casting, Adv. Mater. Sci. Eng., 2014, pp. 1-9.

Mendes M.A.A., Skibina V., Talukdar P., Wulf R., Gross U., Trimis D., Ray S. (2014). Experimental validation of simplified conduction-radiation models for evaluation of effective thermal conductivity of open-cell metal foams at high temperatures, Int. J. Heat Mass Tran., 78, pp. 112-120.

Meyer F. (1991). Un algorithme optimal pour la de ligne de partage des eaux. In : 8 congres AFCET Reconnaissance des Formes et Intelligence Artificielles. Lyon, pp. 847-857.

Müller D.W., Matz A.M., Jost N. (2013). Casting open porous Ti foam suitable for medical applications. Bioinspired, Biomimetic and Nanobiomaterials, 2, pp. 76-83.

Pani M., Taddei F. (2013). The cell Method: Quadratic Interpolation with Tetrahedra for 3D Scalar Fields. CMES: Comput. Modell. Eng. Sci., 94, pp. 279-300.

Plateau J. (1873). Statique Expérimentale et Théorique des Liquides Soumis aux Seules Forces Moléculaires. Vol. 2, Gauthier Villars, Paris, pp. 495. 
Poincaré M.H. (1895). Analysis Situs. Journal de l'école polytechnique, (II) 1, pp. 1-123.

Scheuermann T.M., Kotyczka P., Zanota M.L. Pitault I., Louati H., Maschke B. (2019). Numerical approximation of heat transfer on heterogeneous media. In: Applied Mathematics and mechanics, GAMM, Vienna.

DOI:10.1002/pamm.201900372

Simone AE, Gibson LJ. (1998). Aluminium foams produced by liquid-state processes. Acta. Mater.,46, pp. 3109-3123.

Thomson W., (1887). On the division of space with minimum partitional area. Phil. Mag., 5, pp. 503-514.

Tonti E. (1976). The reason for analogies between physical theories. Appl. Math. Modelling, 1, pp. 37-50.

Tonti E. (2001). A direct discrete formulation of field laws: The cell method, CMES: Comput. Modell. Eng. Sci., 2, pp. 237-258.

Tonti E. (2013). The mathematical structure of classical and relativistic physics, A general classification diagram, Birkhäuser (Springer), New York, p. 514.

Van der Schaft A.J., Maschke B.M., (2013). Port-Hamiltonian Systems on Graphs. SIAM J. Cont. Opt., 51, pp. 906-937.

Veblen O., Whitehead J.H.C. (1932). The foundation of differential geometry. Cambridge Tracts, 29. London: Cambridge Univ. Press.

Vicente J. Brun E. (2009). iMorph. [online] available at: http://imorph.sourceforge.net.

Vicente J. (2017). A 3D image analysis software for cellular material characterisation. iMorph (v3.3) handbook, p. 90.

Vicente J., Topin F., Daurelle J.V. (2006). Open celled material structural properties measurement: from morphology to transport properties. Mater. Trans., 47, pp. 2195-2202.

Volz R.A. (1965). Reticulated polyurethane foams and process for their production, US3171820.

Wang L.C., Wang F. (2001). Preparation of the open pore aluminium foams using investment casting process. Acta Metall. Sinica, 14, pp. 27-32.

Weaire D., Hutzler S. (1999). The Physics of Foam. Clarendon Press, Oxford, p. 246.

Zhao C.Y., Lu W., Tian Y. (2010) Heat transfer enhancement for thermal energy storage using metal foams embedded within phase change materials (PCMs). Sol. Energy, 84, pp. 1402-1412.

Zhao C.Y. (2012). Review on thermal transport in high porosity cellular metal foams with open cells. Int. J. Heat Mass Tran., 55, pp. 3618-3632.

\section{Innex. 1}

To give an inner orientation to p-cell we may proceed as follow, let's consider a simple case of tetrahedron, which is represented by an arrangement of vertices $\left\langle\mathrm{V}_{0}, \mathrm{~V}_{1}, \mathrm{~V}_{2}, \mathrm{~V}_{3}\right\rangle$ of a tetrahedron, which defines an inner orientation of the 3 cell. The latter induces an inner orientation on its faces following the Boundary definition (Franz, 1968):

$$
\partial(S)=\sum_{k=0}^{n}(-1)^{k}\left(V_{0}, \ldots \widehat{V}_{k}, \ldots V_{n}\right)
$$

where $\widehat{V_{k}}$ means that this vertex must be omitted. By applying this definition in the case of the tetrahedron, we can attribute orientations to each face (2-cell).

$$
\partial\left(S_{T}\right)=\sum_{k=0}^{3}(-1)^{k}\left(V_{0}, \ldots \widehat{V_{k}}, \ldots V_{3}\right)=\left(V_{1} V_{2} V_{3}\right)-\left(V_{0} V_{2} V_{3}\right)+\left(V_{0} V_{1} V_{3}\right)-\left(V_{0} V_{1} V_{2}\right)
$$

We can also obtain the orientation on common edges between two adjacent faces following the same rule:

$$
\begin{aligned}
& \partial\left(\partial S_{T}\right)=\partial\left(V_{1} V_{2} V_{3}\right)-\partial\left(V_{0} V_{2} V_{3}\right)+\partial\left(V_{0} V_{1} V_{3}\right)-\partial\left(V_{0} V_{1} V_{2}\right)=\left[\left(V_{2} V_{3}\right)-\left(V_{1} V_{3}\right)+\left(V_{1} V_{2}\right)\right]- \\
& {\left[\left(V_{2} V_{3}\right)-\left(V_{0} V_{3}\right)+\left(V_{0} V_{2}\right)\right]+\left[\left(V_{1} V_{3}\right)-\left(V_{0} V_{3}\right)+\left(V_{0} V_{1}\right)\right]-\left[\left(V_{1} V_{2}\right)-\left(V_{0} V_{2}\right)+\left(V_{0} V_{1}\right)\right]=0}
\end{aligned}
$$

The orientation induced on their common element are opposite according to the Möbius law of edges (Klein 1948). 